\title{
POSTNATAL IMPLICATIONS FOR PRENATAL DIAGNOSED TETRALOGY OF FALLOT
}

\author{
Georgiana Nicolae $^{1,2}$, Cristina Filip ${ }^{1,2}$, Adriana Diaconu ${ }^{1,3}$, Alin Nicolescu ${ }^{1,2}$, \\ Constantin Arion ${ }^{1,3}$ \\ 1 "Carol Davila" University of Medicine and Pharmacy, Bucharest \\ 2 "Marie Curie" Emergency Hospital for Children, Bucharest \\ 3 "Fundeni" Clinical Institute, Bucharest
}

\begin{abstract}
Over the last few years, prenatal diagnosis of congenital heart diseases experienced an important development. Overall survival of patients with congenital heart disease was improved in the last decade due to the advanced diagnostic methods and progression of cardiovascular surgical procedures in this field.

Due to the significant increase of the long-term survival rate, currently, in most developed countries, there are departments dedicated to monitoring the adults with congenital heart malformation due to the particularities and special needs of this type of patients, compared with the regular cardiac pathology of adults.

This article proposes a review of existing data at this moment related to the prenatal and postnatal diagnosis and their impact on the evolution regarding the most common cyanotic congenital malformation - Tetralogy of Fallot. In the following pages, we is emphasize the importance of knowing the precise fetal pathology for early determination of medical behavior in the postnatal period for the best care of these children.
\end{abstract}

Keywords: congenital heart malformation, Tetralogy of Fallot, prenatal diagnosis

The incidence of congenital heart disease in Romania is 7.5/1,000 live births (1).

Most heart abnormalities can be identified from the $12^{\text {th }}$ week of pregnancy at an ultrasound examination performed by an experienced physician with performant equipment [eg. Tetralogy of Fallot, ventricular hypoplasia (the left or right), ventricular septal defect]. At 16 to 18 weeks of pregnancy we can observe: atrial septal defect, valvular stenosis, transposition of great vessels (2).

Tetralogy of Fallot (TOF) is the most common cyanotic heart malformation, with an incidence of 32.6 per 100,000 live births again. In this type of malformation with complete surgical correction, the short and long-term prognosis is influenced by the anatomic characteristics that can be diagnosed prenatally, the surgical technique, and the presence of other associated abnormalities (3).

The original description of TOF included four anomalies: a large septal ventricular defect (VSD), obstruction in the right outflow tract (RVOT), right ventricular hypertrophy (RVH) and dextroposition of the aorta (Fig. 1). Nowadays, only two anoma- lies have are considered necessary for the diagnosis: a VSD large enough to equalize the pressure between the ventricles and the obstruction of the RVOT.

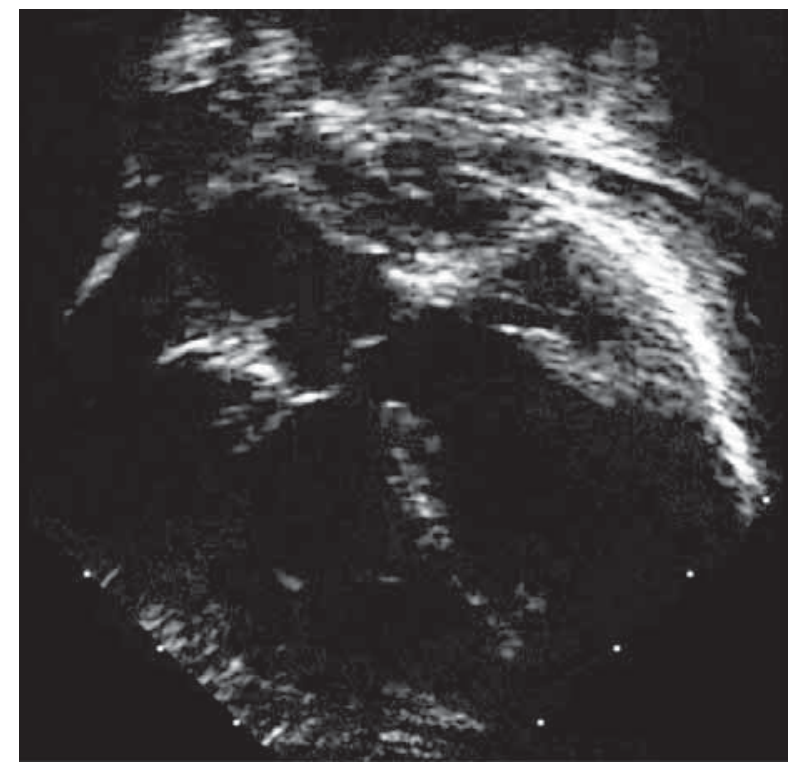

FIGURE 1. Modified apical 4-chamber view demonstrating the overriding aorta above the crest of the ventricular septum and a large ventricular septal defect 
The obstruction of RVOT is mostly presented as infundibular stenosis (45\%). The obstruction is rarely al the valve level (10). A combination between infundibular and valvular stenosis seems to be quite frequent (30\%) (Fig. 2).

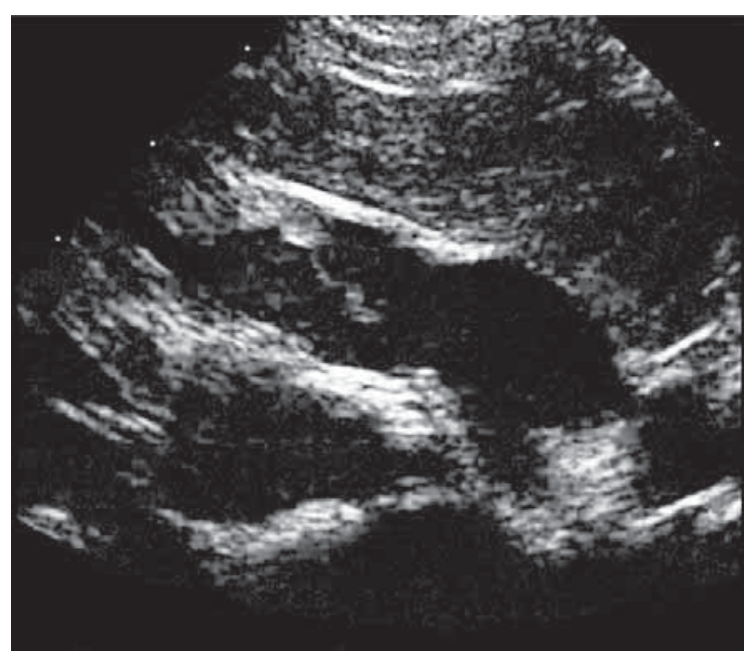

FIGURE 2. High left parasternal long-axis view angled leftward showing subpulmonary infundibular hypertrophy, mildly hypoplastic pulmonary valve annulus

In the most severe form of this anomaly, the pulmonary valve is atretic (4) (Fig. 3).

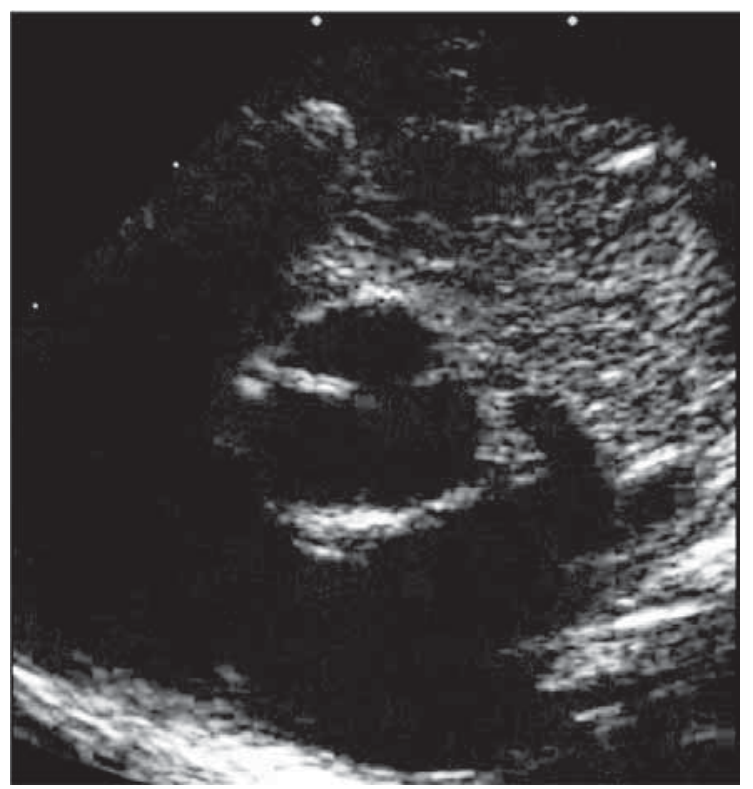

FIGURE 3. Parasternal short-axis imaging in an infant with tetralogy of Fallot and long-segment pulmonary atresia

Pulmonary valvular annulus and main pulmonary branches are variable hypoplastic in most patients. Pulmonary artery branches are usually small, although severe hypoplasia is rare. The stenosis of the origin of the pulmonary branches, in particular the left pulmonary artery is also common. Occasionally, especially in severe cases of TOF, there are systemic - pulmonary collateral arteries necessary to provide the lungs blood flow.
In $25 \%$ of cases, the right aortic arch is present, with possible symptoms of vascular ring. In about $5 \%$ of patients with TOF, anomalous coronary arteries are present. The most common abnormality is the presence of a descending branch from the right coronary artery, the artery that runs over RVOT not allowing a surgical incision in the region. Common atrioventricular canal-complete form occurs in about $2 \%$ of cases, with a much greater frequency among patients with Down syndrome. In these patients, VSD has an "inlet" component, complementary to the "outlet" large component associated with atrioventricular canal (5).

Poon et al. reported their experience with the fetal diagnosis of TOF at a mean gestational age of 20.6 weeks.

Associated extracardiac anomalies can occur in up to approximately $30 \%$ of affected infants and children, and the incidence can be as high as 50 $60 \%$ in fetal TOF.

Appropriate prenatal evaluation and counseling for TOF require careful high-level screening for noncardiac anomalies and chromosomal anomalies.

The diagnosis of TOF is often not obvious in the 4-chamber view. The LV outflow tract view or the 5-chamber view demonstrate aortic override and on color flow mapping flow is directed from both ventricles into the aorta. The basal short-axis view demonstrates the deviation of the conal septum, malalignment VSD and subpulmonary stenosis.

The 3-vessel view can demonstrate vessel size discrepancy, with the aorta being larger than the pulmonary artery. The flow in the ductus arteriosus would be retrograde in the case of severe pulmonic stenosis or atresia. Aortic arch sidedness and arch anomalies can be evaluated in the 3-vessel axial view, and the transmission qualities of the unexpanded fetal lungs can facilitate the prenatal diagnosis of vascular rings. Serial prenatal echocardiography is recommended, primarily to reassess for progressive subpulmonary stenosis and/or progressive pulmonary artery hypoplasia (6).

Fetuses with tetralogy of Fallot may present with only a ventricular septal defect and aortic septal override by prenatal ultrasound examination (Fig. 4). Pulmonary artery stenosis is not always present at initial ultrasound examination, but this finding can develop or worsen during pregnancy. Furthermore, a normal aortic diameter does not exclude the tetralogy of Fallot. Infant survival appears to be favorable in the absence of other major structural or chromosomal anomalies (7). 


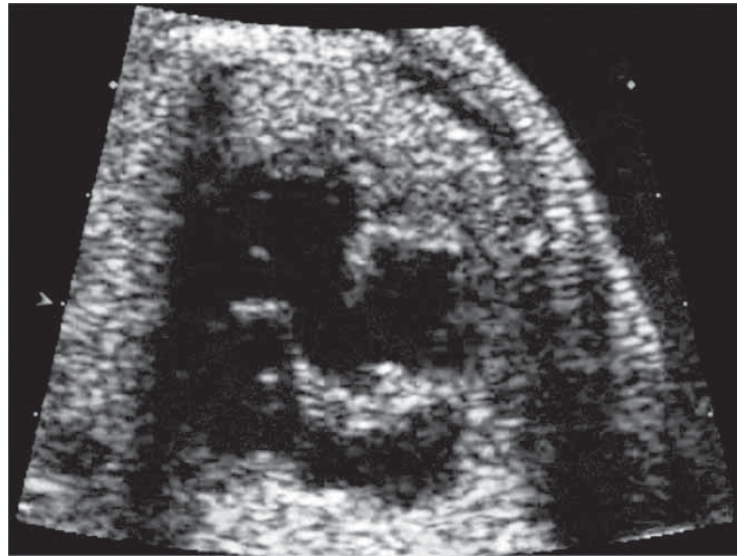

FIGURE 4. Fetal echocardiogram showing the long-axis of the left ventricle, malalignment conoventricular septal defect and aortic override

In a retrospective study conducted in California over a 12 year period (1985-1997) on the conotruncal anomalies (DORV, TOF, malposition of the great arteries, transposition of great arteries, pulmonary atresia, DSV, common arterial trunk), were found 2,470 prenatal echocardiographies. 61 fetuses with conotruncal abnormalities were confirmed. The mean age at diagnosis was 24.5 weeks of gestation and concordance with the prenatal diagnosis was $77 \%$. It has been found that from the conotruncal anomalies, Tetralogy of Fallot, excluding those with absent pulmonary valve, had a better prognosis. This study demonstrates that conotruncal anomalies can be diagnosed by prenatal echocardiography with a relatively high degree of accuracy. The clinical outcome of the fetuses with conotruncal anomalies is poor, even without major extracardiac anomalies, except for the fetuses with tetralogy of Fallot. Their survival had been $75 \%$ in the neonatal period (8).

In 2016, was published a meta-analysis that included 36 articles from international databases Medline and Embase. It has followed the evolution of patients with TOF in the postnatal period, the association with genetic syndromes and concordance between prenatal and postnatal ultrasound data. It was concluded that the survival rate in the late neonatal period of patients diagnosed prenatally with atresia or absent pulmonary valve is significantly lower compared to those with TOF with pulmonary stenosis. It also revealed that genetic anomalies and associated malformations were more common in TOF with pulmonary atresia or absent pulmonary valve against TOF with pulmonary stenosis. For the TOF with pulmonary valve atresia (which is currently the most accepted the "atresia of the pulmonary valve with VSD"), included in ducto-dependent cyanotic congenital heart malformation, correct prenatal diagnosis requires treatment with prostaglandin immediately after birth. $11.7 \%$ of these fetuses have a genetic syndrome, chromosome abnormalities were found in $8.3 \%$ of cases and $6.7 \%$ associate other organ malformations. Only $73.3 \%$ of these children have no other associated anomalies. The most common genetic syndrome associated with this type of TOF (8-23\% of patients) is DiGeorge SDR/SDR velocardiofacial (22q11.2 deletion). Also, $10 \%$ of patients with $22 q 11.2$ deletion have TOF with pulmonary valve atresia (this extreme form of TOF is apparently more common in genetic syndromes than other forms of TOF). Also, in fetuses with TOF and pulmonary valve atresia, associated cardiac anomalies were more frequent than in fetuses with common forms of TOF - commonly associated right aortic arch, aberrant subclavian artery origin, aorto-pulmonary collateral.

TOF with absent pulmonary valve represents $3-6 \%$ of all patients with TOF; the pulmonary valve is absent or is only rudimentary. This variant is associated with severe aneurysmal dilatation of the pulmonary artery trunk and branches that can lead to airway compression with severe respiratory failure at birth. Usually, these fetuses have an associated degree obstruction in RVOT. An important feature of this form of Fallot is that ductus arteriosus is almost always absent. $50 \%$ of these patients had a right aortic arch (9).

Prenatal echocardiographic diagnosis of CHD experienced an important development in recent years. If in 1997 the concordance between prenatal and postnatal diagnosis in the most common types of CHD was $77 \%$, now it has risen to over $90 \%$.

Tetralogy of Fallot shows a broad spectrum of clinical forms. Particularities of each type of TOF can now be studied closely by a detailed ultrasound evaluation (10).

The correct fetal diagnosis of a severe form of TOF (with atresia/pseudoatresia of the pulmonary valve or form with severe pulmonary artery hypoplasia) requires birth in a maternity with maximum intensive care possibilities, with direct access to a service of pediatric cardiology and pediatric cardiovascular surgery; also, neonatologists will initiate therapy with prostaglandin i.v. to maintain the ductus arteriosus open until the surgical correction (palliative in a first stage) is made in the neonatal period.

For the fetuses with the diagnosis of TOF with absent pulmonary valve, a neonatologist will be informed; in the case of infants who experience severe respiratory failure at birth (by airway com- 
pression, aneurysmal dilatation of the trunk and pulmonary artery branches), it will be necessary immediate respiratory prosthesis and positive pressure ventilation to maintain an open airway. Babies born with clinical symptoms will require surgery in the neonatal period, but it involves a high operational risk and postoperative results in many cases are unsatisfactory.

\section{CONCLUSIONS}

Correct fetal diagnosis of TOF requires great care in identifying cardiac and extra-cardiac associated anomalies and genetic investigations to detect chromosomal anomalies. Advising the parents can be done realistically presenting the full clinical and genetic context of the child, the possibilities of surgical correction and appreciation on postnatal prognosis. It's important that the parents will understand that heart disease benefits from total correction if it's an isolated condition, with favorable prognosis in the long term. Unfavorable long-term prognosis is related mainly to the presence of genetic syndromes and other associated malformations.

Is also of great importance the assessing of dynamics of the fetus after the initial diagnosis, given the possibility of worsening the degree of obstacle in RVOT, as well as hypoplasia of pulmonary valve annulus, pulmonary artery trunk and branches, so near the time of birth, severity of the disease to be appreciated as well as possible.

\section{REFERENCES}

1. Eugen Ciofu, Carmen Ciofu. Esențialul în Pediatrie, $2^{\text {nd }}$ edition, 2001

2. Jack Rychik, Zhiyun Tian, Denise D. Donaghue, The Cardiac Center at The Children's Hospital of Philadelphia, ISUOG Congress U.S.A., 2011

3. Hoffman J.I., Kaplan S. The incidence of congenital heart disease. J Am Coll Cardiol 2002; 39:1890-900.

4. Pierpont M.E., Basson C.T., Benson D.W. Jr et al. Genetic basis for congenital heart defects: current knowledge: a scientific statement from the American Heart Association Congenital Cardiac Defects Committee, Council on Cardiovascular Disease in the Young: endorsed by the American Academy of Pediatrics. Circulation 2007; 115:3015-38.

5. Geva T., Ayres N.A., Pac F.A., Pignatelli R. Quantitative morphometric analysis of progressive infundibular obstruction in tetralogy of Fallot. A prospective longitudinal echocardiographic study. Circulation 1995; 92:886-92.

6. Wyman W. Lai, Luc L. Mertens, Meryl S. Cohen, Tal Geva. Echocardiography in Pediatric and Congenital Heart Disease: From Fetus to adult, 2009.

7. https://www.ncbi.nlm.nih.gov/pubmed/17405110

8. https://www.ncbi.nlm.nih.gov/pubmed/7675384

9. http://content.onlinejacc. org/article. aspx?articleid $=1125748$

10. https://www.ncbi.nlm.nih.gov/pubmed/27022172 